\title{
Effects of contextual elaboration on face recognition
}

\author{
NANCY H. KERR \\ Emory University, Atlanta, Georgia 30322, and \\ Cognition Research Laboratory, Georgia Mental Health Institute, Atlanta, Georgia 30306
}

and

EUGENE WINOGRAD

Emory University, Atlanta, Georgia 30322

\begin{abstract}
Four experiments were conducted to test the effects of an elaborated verbal encoding context on face recognition. The verbal context consisted of short descriptive phrases about the person shown, and the degree of elaboration was manipulated by varying the number of such phrases across faces. In all four experiments, subjects initially studied faces either alone or accompanied by one or more descriptive phrases. The first two experiments required subjects to recognize faces unaccompanied by the original phrases, the third required phrase recall as well as face recognition, and the fourth experiment tested face recognition both with and without the reinstatement of the original verbal encoding context. All four experiments provided evidence that subjects were more likely to recognize a face when they had received information about the person than when they had not. An interaction between encoding and retrieval was found in Experiment 4 such that elaboration facilitated face recognition more when the encoding context was reinstated than when it was not. The results demonstrate that verbal elaboration of a meaningful visual stimulus can facilitate recognition memory.
\end{abstract}

Recent theory and research suggest that the degree of elaboration a stimulus receives at encoding plays a major role in determining how well it will be remembered (e.g., Anderson \& Reder, 1979; Craik \& Tulving, 1975; Fisher \& Craik, 1980; Klein \& Saltz, 1976). The term "elaboration" is used to describe the encoding of additional relevant information about a stimulus and its encoding environment to establish a richer, more extensive memory trace. Craik and Tulving (1975), for example, found that words presented in the context of complex sentences were better recalled than were those presented with simpler sentences, and Klein and Saltz (1976) found that subjects recalled words better when they had rated them on two uncorrelated semantic dimensions rather than on one. Elaboration generally refers to the quantitative amount of information encoded along with a stimulus. It is widely recognized that it is difficult to separate the amount of elaboration from the distinctiveness or uniqueness of the resultant memory trace (Craik \& Jacoby, 1979; Winograd, 1981). Memory for familiar verbal stimuli has been shown to rely on the amount and distinctiveness of elaboration and on the degree of con-

This research was supported in part by NIMH Grant 32063. We gratefully acknowledge the assistance of Vaughan Church in the data collection and analysis. Requests for reprints should be sent to Nancy H. Kerr, Cognition Research Laboratory, GMHI, 1256 Briarcliff Road, N.E., Atlanta, Georgia 30306. textual overlap at encoding and test (Craik \& Tulving, 1975; Fisher \& Craik, 1980; Klein \& Saltz, 1976).

Theoretically, the degree of elaboration of the encoding context may also be expected to affect memory for novel nonverbal stimuli, such as unfamiliar faces, which are potentially meaningful. Indeed, Anderson and Reder (1979) have argued that it is precisely this susceptibility of faces to meaningful elaboration that makes them more memorable than equally complex visual stimuli such as snowflakes (Goldstein \& Chance, 1970). Furthermore, the susceptibility of faces to contextual influence has been demonstrated empirically. Winograd and Rivers-Bulkeley (1977), for example, showed that the reinstatement of a visual encoding context (another face) during the recognition test increased face recognition, and Watkins, Ho, and Tulving (1976) obtained similar results using a descriptive verbal context. These studies, however, focused on the effects of reinstatement of the encoding context at retrieval and did not manipulate the encoding context systematically. Chance and Goldstein (1976) found a small positive effect of selfgenerated verbal labels on face recognition, but only when instructions required that verbal labels describe memorable features of the face itself.

The present research was designed to test the possibility that verbal elaboration at encoding in the form of information about the person shown (e.g., "he is a concert pianist; he is divorced") will facilitate subsequent 
recognition of unfamiliar faces. The questions addressed are as follows. First, as Fisher and Craik (1980) point out, there is reason to question whether elaborative effects can be demonstrated in recognition memory at all. Aside from Fisher and Craik's (1980) study, the entire literature on elaboration and memory deals with recall. Fisher and Craik found no effects of elaboration on word recognition unless the original encoding context was reinstated, and they cite an unpublished study by Tulving that found no effects of sentence complexity on word recognition. In this study, we examine the effects of varying degrees of elaboration on facial recognition both when the encoding context is and when it is not reinstated. Second, we ask whether verbal elaboration of a visual stimulus, a face, affects memory. Bahrick (1979) has suggested that elaboration of unfamiliar nonverbal stimuli should be based on the physical characteristics of the items themselves rather than on their encoding environments. Bahrick attributes the context effects for unfamiliar faces found by Watkins et al. (1976) to special pretraining to attend to the context. In the present study, we test whether verbal elaboration facilitates face recognition under conditions in which subjects have had no special training and have been informed only that face recognition will be tested. The elaborative statements deliberately omitted any reference to physical features of the faces. Third, a reliable finding in the literature is that memory for faces is enhanced following judgments about character traits such as honesty or friendliness (see Winograd, 1978, for a review). An explanation in terms of elaboration and distinctiveness has been offered by Winograd (1981). Essentially, Winograd argues that such trait judgments induce a more extensive scan of the face, which maximizes the number of features encoded and, consequently, the probability of sampling a distinctive feature. However, this argument is only inferential, since no independent evidence of broader feature sampling of the face has been presented. In the present research, degree of elaboration is identified with the amount of information accompanying each face at study, and the problem of circularity is thereby diminished.

In Experiment 1, faces were accompanied by zero, one, two, or three short descriptive statements at study and were tested without context. It was expected that higher recognition accuracy would be associated with a greater number of elaborative statements.

\section{EXPERIMENT 1}

\section{Method}

Subjects. The subjects were 24 (11 female, 13 male) young adults who responded to bulletin board notices on college campuses and were paid for their participation.

Materials and Design. The faces in all of these experiments were black-and-white $35-\mathrm{mm}$ slides of head-and-shoulders pictures of adults of various ages taken from a British theatrical casting directory. In this experiment, only males were shown. Seventy-two faces were presented for study, but the initial and final four faces were not tested. Copies of the remaining 64 original faces were randomly mixed with 64 new faces for the recognition test.

One hundred and eight short descriptive phrases, taken from or modeled after those used by Watkins et al. (1976), were presented along with the faces during study trials. The phrases described personality characteristics, hobbies, professions, and so on. Some sample phrases were "he's an alcoholic," "he's a vegetarian," "he smokes cigars," and "he's a psychiatrist." Each face at presentation was accompanied by zero, one, two, or three of these descriptive phrases, and each subject saw 16 faces in each elaboration condition. The faces always were presented in the same order, but phrases accompanied faces in 12 different random assignments such that each face appeared in each of the four conditions an equal number of times and each phrase appeared in each of the three elaboration conditions equally often. The phrases that appeared together in the two-and threephrase conditions were randomly selected, but contradictory or incongruous pairings (e.g., two occupations for one person) were not used. Two subjects received each of the 12 phrase orders.

Procedure. Subjects were tested singly or in pairs. Included in the instructions was this statement: "I would like you to study the faces carefully so that you will be able to recognize them when they are presented to you in a later part of the experiment. You should also pay careful attention to the things that $I$ tell you about the person." These instructions were common to all the experiments reported here. Slides were presented by a Kodak Carousel projector at a rate of $8 \mathrm{sec} / \mathrm{slide}$ for both study and test. The experimenter began to read the descriptive phrase(s) as soon as each slide appeared, and in all cases the description was completed within the $8 \mathrm{sec}$ interval. In the zero-phrase (no elaboration) condition, the experimenter read the words "no information." Subjects were tested for recognition of faces unaccompanied by phrases. Subjects were instructed to judge each face as "old" or "new" and to indicate on a 3-point scale how confident they were of each judgment.

\section{Results and Discussion}

The proportions of hits for the four conditions are presented in Table 1 , along with mean confidence ratings weighted for accuracy. These confidence scores were calculated only for old items on a 6-point scale, with the highest value of 6 assigned to hits made with maximum confidence and the lowest value of 1 to misses judged with maximum confidence. The overall false alarm rate was .16. Due to the within-subjects design, the false alarms could not be partitioned.

Analyses of variance were significant both for hits $[F(3,69)=8.43, \mathrm{MSe}=3.20, \mathrm{p}<.01]$ and for confidence scores $[\mathrm{F}(3,69)=10.12, \mathrm{MSe}=.20, \mathrm{p}<.01]$. Newman-Keuls pairwise comparisons for hits were significant for each of the phrase conditions compared to the no-elaboration condition and for one phrase compared to two phrases. The same comparisons were significant for the confidence scores, and the compari-

Table 1

Mean Proportions of Hits and Mean Confidence Ratings for the Elaboration Conditions of Experiment 1

\begin{tabular}{lcccc}
\hline & \multicolumn{5}{c}{ Elaboration (Number of Phrases) } \\
\cline { 2 - 5 } $\begin{array}{c}\text { Dependent } \\
\text { Measure }\end{array}$ & 0 & 1 & 2 & 3 \\
\hline Hit Rate & .65 & .73 & .80 & .78 \\
Confidence & 4.25 & 4.57 & 4.88 & 4.84 \\
\hline
\end{tabular}


son of one phrase to three phrases was significant as well.

The results support the hypothesis that facial recognition increases as the degree of verbal elaboration of the face increases. However, an alternative explanation for the results attributable to the within-subjects design is as follows. It is possible that subjects used the presence of information about a face as an indication of its importance as a stimulus item. Faces for which there was no information may have received less scrutiny because they were judged to be less important than the others. To test this possibility and the reliability of the results of Experiment 1, our second experiment employed similar materials in a between-subjects design with the same basic procedure.

\section{EXPERIMENT 2}

\section{Method}

Subjects. Forty-eight (20 female, 28 male) Emory University undergraduates participated for credit toward a psychology class requirement.

Materials and Design. The faces and short descriptive phrases were selected from the same sources as those used in Experiment 1. Three degrees of elaboration were presented in this experiment, zero phrases, one phrase, or two phrases. All subjects viewed 56 faces at presentation, the first and last 4 of which were not tested. Copies of the remaining 48 faces were mixed randomly with 48 new faces for the recognition test. Slides appeared in a constant order. Four different random pairings and orders of the 96 descriptive phrases were assembled for the two-phrase condition, as were four random orders of single phrases for the one-phrase condition. Four subjects were exposed to each phrase order.

Procedure. Subjects were randomly assigned to conditions and were tested singly or in small groups. Slides were presented at an 8 -sec rate by a Kodak Carousel projector. The experimenter remained silent during presentation in the zero-phase condition and read the phrases as in Experiment 1 for the other two conditions. The recognition test procedure was the same as that for Experiment 1.

\section{Results and Discussion}

The proportion of hits and false alarms for the three conditions are presented in Table 2 along with mean $\mathrm{d}^{\prime}$ and confidence difference scores. Confidence difference scores were calculated by converting all answers to the 6-point scale described in Experiment 1 and then subtracting each subject's mean score for new items from the mean score for old items. Analyses of variance were computed for the $\mathrm{d}^{\prime}$ and confidence difference scores. Both were significant $[\mathrm{F}(2,45)=3.43, \mathrm{MSe}=.30$, $\mathrm{p}<.05$ for $\mathrm{d}^{\prime} ; \mathrm{F}(2,45)=4.54, \mathrm{MSe}=.36, \mathrm{p}<.05$ for the confidence difference scores]. With both measures, Newman-Keuls pairwise comparisons were significant only for the difference between the no-elaboration and one-phrase conditions. Neither the hit nor false alarm measure proved significant.

The results replicate Experiment 1 in finding that the presence of elaborative verbal information about a face at encoding increases the likelihood that it subsequently
Table 2

Mean Hits, False Alarms, d', and Confidence Difference Scores for Experiment 2

\begin{tabular}{lccc}
\hline \multicolumn{1}{c}{ Dependent Measure } & \multicolumn{3}{c}{$\begin{array}{c}\text { Elaboration } \\
\text { (Number of Phrases) }\end{array}$} \\
\cline { 2 - 4 } & 0 & 1 & 2 \\
\hline Hit Rate & .77 & .78 & .81 \\
False Alarm Rate & .21 & .11 & .18 \\
d' & 1.67 & 2.17 & 1.86 \\
Confidence Difference Score & 2.31 & 2.95 & 2.64 \\
\hline
\end{tabular}

will be recognized, but they suggest an all-or-none rather than a graded function relating elaboration and memory. The failure to find reliable effects for the number of elaborative statements may be due either to subjects' failure to encode all of the elaborative statements or to our failure to provide an adequate testing environment to reveal the effects of elaboration (Fisher \& Craik, 1980). Experiment 3 explores the former possibility, and Experiment 4, the latter.

The possibility that subjects are not encoding multiple phrases is raised by the equivalence of recognition performance in the two- and three-phrase conditions in Experiment 1 and in the one- and two-phrase conditions in Experiment 2. The time constraints of the experimental situation may limit the amount of information a subject is able to encode, and subjects may, on the average, remember only one descriptive phrase per face regardless of the number presented. Experiment 3 assessed whether subjects actually encode more than one experimental phrase per face by testing phrase recall as well as face recognition.

The within-subjects design of Experiment 1 was repeated for Experiment 3 in an attempt to replicate the finding of better recognition for faces accompanied by two or three phrases than for those accompanied by one. If the replication succeeded, the recall data could help to clarify whether the better recognition was due to the encoding of more phrases when two or three were presented or to the fact that when subjects heard more phrases there was a greater likelihood that one of the phrases would seem appropriate for the face and would therefore be more easily encoded with the face. Research has shown that for words, at least, increasing the congruence of a stimulus and its encoding context increases memory performance (Craik \& Tulving, 1975;Schulman, 1974).

\section{EXPERIMENT 3}

\section{Method}

Subjects. Sixteen ( 2 female, 14 male) Emory University undergraduates participated for credit toward a psychology class requirement.

Materials and Design. The faces and phrases were the same as those used in Experiment 1, and the within-subjects design differed only in that 36 rather than 64 new slides were included 
in the recognition test. The three-phrase elaboration condition was included. Eight of the phrase orders from Experiment 1 were used, with two subjects exposed to each.

Procedure. All subjects were tested individually using the study procedure and instructions from Experiment 1 . During the face recognition test, instead of being asked to rate confidence, subjects were asked to write down as much of the verbal information as they could remember about each of the faces they recognized from the study trial, including the words "no information" for the faces that had been accompanied by those words originally. Subjects were told that approximately twothirds of the faces in the test would be familiar ones. The recognition test was individually paced to allow each subject as much time as necessary to recall the phrases.

\section{Results and Discussion}

The proportion of hits on the recognition test for each elaboration condition is shown on the first row of Table 3. The proportion of false alarms was .14. The analysis of variance for hits was significant $[F(3,45)=$ $3.16, \mathrm{MSe}=2.02, \mathrm{p}<.05]$. The one-phrase condition was significantly different from the no-elaboration condition by Newman-Keuls pairwise comparisons. The results once again indicate that the presence of at least one descriptive phrase at encoding increases face recognition, but we did not find greater effects for two and three phrases than for one.

The recall data also are summarized in Table 3. Because of the equivalence of the one-, two-, and threephrase conditions on the face recognition test, these recall data are useful only in providing some indication of the extent to which additional information was encoded. The mean numbers of faces for which at least one correct phrase was recalled were similar across the one-, two-, and three-phrase conditions, suggesting that increasing the number of statements about a face did not increase the probability that a "congruent" and consequently more memorable statement would appear with a face in the two- and three-phrase conditions. Subjects did recall more total phrases in the two- and threephrase conditions than in the one-phrase condition, and they recalled combinations of phrases about as often as they recalled isolated phrases. This illustrates that at

Table 3

Mean Proportions of Hits and Mean Recall Scores for Experiment 3

\begin{tabular}{|c|c|c|c|c|}
\hline \multirow[b]{2}{*}{ Dependent Measure } & \multicolumn{4}{|c|}{$\begin{array}{c}\text { Elaboration } \\
\text { (Number of Phrases) }\end{array}$} \\
\hline & 0 & 1 & 2 & 3 \\
\hline $\begin{array}{l}\text { Hit Rate } \\
\text { Faces With at Least One Phrase Recalled } \\
\text { Phrases Correctly Recalled* } \\
\text { Phrases Correctly Recalled Alone } \\
\text { Phrase Pairs Correctly Recalled } \\
\text { Phrase Triplets Correctly Recalled }\end{array}$ & .69 & $\begin{array}{r}.79 \\
2.56 \\
2.56 \\
2.56\end{array}$ & $\begin{array}{r}.76 \\
2.56 \\
3.69 \\
1.44 \\
1.12\end{array}$ & $\begin{array}{r}.75 \\
2.19 \\
3.75 \\
1.06 \\
.69 \\
.44\end{array}$ \\
\hline
\end{tabular}

The maximum possible score was 16 in the one-phrase condition, 32 in the two-phrase condition, and 48 in the three-phrase condition. least some of the additional descriptive information in the two- and three-phrase conditions was actually encoded, although the low overall recall rates would suggest that recall of phrases has little influence on the face recognition decision. Incorrectly recalled phrases were never novel phrases but, instead, were inappropriately applied phrases from the presentation list. Subjects misapplied phrases to an average of 4.5 old faces (9\% of correct recognition faces) and .75 new faces (15\% of the false alarm faces). A subject rarely repeated the same phrase for more than one face, but some subjects reported recognizing a previous error in phrase attribution when they saw the appropriate face (e.g., "It wasn't the other one that liked Mozart, it's this one"). Overall, these errors appeared to reflect associative confusions rather than confabulation or pure guessing.

The results of the first three experiments, then, show a consistent facilitative effect on face recognition for one descriptive elaborative phrase, but inconsistent effects for an increased number of phrases. The explanation for these results may lie in the absence of an appropriate retrieval context. Thus, Fisher and Craik (1980) found that the effects of elaborative encoding for words were not apparent on a recognition test unless there was reinstatement of the original elaborative context at test. They concluded that, for recognition memory, "the mere addition of elaborative information at encoding is not sufficient to improve retention. Rather it alters only the potential memorability of an event, and reinstatement of the initial encoding context by one means or another is also necessary before the beneficial effects are observed" (Fisher \& Craik, 1980, p. 403). By this line of reasoning, our failure to find increasing recognition for faces with increasing amounts of elaborative information in Experiments 2 and 3 may have been the result of not providing elaborated contexts at both presentation and test. It should be noted that Fisher and Craik (1980) did not include a noelaboration, or word-only, condition comparable to our condition in which the faces were unaccompanied by descriptions.

Experiment 4 was therefore designed to look for an interaction between elaboration and reinstatement of the encoding context at retrieval in face recognition. The results of our first three experiments, in light of Fisher and Craik's (1980) work, suggest that the presence of elaborative phrases at encoding will enhance face recognition overall, but to a greater extent when the phrases are present at both encoding and retrieval.

\section{EXPERIMENT 4}

\section{Method}

Subjects. Sixty-four ( 31 female, 33 male) Emory University undergraduates participated for credit toward a psychology course requirement.

Materials and Design. In this study, pictures of both adult males and females were included. At study, the 45 male and 
45 female experimental faces, randomly mixed, were preceded by 10 and followed by 10 untested faces. Copies of the 90 study faces were each presented at test next to a previously unseen face of the same sex for a two-alternative forced-choice recognition test. The order at test and the left-right placement of old and new slides were randomly determined.

One hundred and ten short descriptive phrases, similar to those in Experiments 1, 2, and 3, but potentially applicable to either a male or female, were composed. Each face at presentation was accompanied by zero, one, or two of these phrases. Each subject encountered 30 test faces at each degree of elaboration. Faces appeared in a constant order, but phrases were presented in three different random orders, with each face appearing once in each elaboration condition. Thirty subjects heard the original study phrase or phrases with the faces at test; 34 did not. Elaboration was a within-subjects factor, and the presence or absence of the phrases at test was a between-subjects factor.

Procedure. Subjects participated in groups of three or four. The experimenter read the phrases at presentation as in Experiments 1 and 3 , and the slides were presented at an $8-\sec$ rate. For the forced-choice recognition test, same-sex pairs of one old and one new face were shown simultaneously on the screen, and subjects were asked to indicate whether they believed the face on the right or the left was one they had seen before. In the context-present group, subjects heard the phrase(s) associated with the old face during the presentation of each test pair. Subjects in the no-context condition were not presented with any of the phrases at test, as in the previous experiments.

\section{Results and Discussion}

The proportions of correct choices in each elaborationcontext condition are presented in Table 4 . An analysis of variance of these data gave significant outcomes for elaboration $[\mathrm{F}(2,124)=40.79, \mathrm{MSe}=.003, \mathrm{p}<.01]$ and for the Elaboration by Context interaction $[\mathrm{F}(2,124)$ $=17.91, \mathrm{MSe}=.003, \mathrm{p}<.01]$. Planned comparisons showed that one- and two-phrase elaboration was significantly better than no elaboration $[F(1,124)=$ $39.88, \mathrm{MSe}=.003, \mathrm{p}<.001]$ and that context reinstatement produced better recognition than no context for both the one-phrase $[\mathrm{F}(1,126)=20.42, \mathrm{MSe}=.004$, $\mathrm{p}<.001]$ and two-phrase $[\mathrm{F}(1,126)=6.68$, MSe $=$ $.003, \mathrm{p}<.05]$ conditions. Thus, elaboration at encoding enhanced face recognition in general, but the enhancement was greater when the descriptive information was reinstated at test.

These results may be compared with those of Watkins et al. (1976). Column 2 of Table 4 represents a replication of their Experiment 3, in which faces were accompanied at study by one descriptive statement and were then shown in a two-alternative forced-choice test either accompanied by the study context or by no

Table 4

Mean Proportions of Correct Recognition for Experiment 4

Elaboration (Number of Phrases)

\begin{tabular}{llll} 
& & \multicolumn{3}{c}{ Test Condition } & 0 & 1 & 2 \\
\cline { 2 - 4 } No Context & .82 & .85 & .88 \\
Context Present & .82 & .93 & .92 \\
\hline
\end{tabular}

context. Recognition was higher by $8 \%$ in their study when context was reinstated, the same difference reported in Table 4 in the present study. The present study departs from that of Watkins et al. in varying the amount of context presented at study and test.

\section{GENERAL DISCUSSION}

The results of the four experiments may be summarized as follows. First, it is easier to recognize the face of a person about whom you have been given some personal information than a face you have examined without any accompanying information. Second, if you have been given personal information about a face, you are more likely to recognize the face later if the personal information is repeated with it. The former result is shown most clearly and consistently across experiments when the information is in the form of a single phrase. Although the first experiment did show a significant effect for the amount of information (one phrase vs. two), the effect in the subsequent experiments was neither consistent in direction nor significant. The inconsistent effects of increased information may be attributable in part to constraints of the experimental situation. Specifically, the reading of three phrases generally required the full $8 \mathrm{sec}$ of slide presentation and may have allowed insufficient time for encoding the information. This encoding difficulty may have been magnified by the fact that phrases were thematically unrelated to each other, making them difficult to integrate elaboratively. The importance of the integration of facts in memory has been demonstrated in a variety of experimental tasks (e.g., Clifton \& Slowiaczek, 1981; Reder \& Anderson, 1980). Klatzky, Martin, and Kane (1982) also have demonstrated that congruence or appropriateness of a verbal label (an occupation) to a face may influence subsequent recognition memory. Verbal elaboration of a face, then, facilitates memory, but some questions remain about the shape of the function relating amount of elaboration to recognition memory.

With respect to the issue raised by Fisher and Craik (1980) as to whether elaboration effects are restricted to recall, the answer is clearly no. This research extends their conclusions by showing that elaboration is beneficial to recognizing faces as well as words. Furthermore, the interaction between elaboration and context reinstatement in Experiment 4 supports Fisher and Craik's argument that the effects of elaboration in recognition memory are mediated by retrieval factors, in particular, the reinstatement of context. However, the results of the four studies taken together do not support the strong version of the reinstatement hypothesis argued by Fisher and Craik, namely, that no effect of elaboration will be found in recognition memory unless the encoding context is reinstated. In all of the studies reported here, an effect of elaboration is apparent even when the 
encoding context was not reinstated at test. Fisher and Craik clearly have allowed for such a result, since they remark that "the effects of elaboration will be found with single items only in cases in which the item and its elaborating context are well integrated, and therefore presentation of the item alone will serve to re-integrate the encoding context" (1980, p. 403). One can readily apply this observation to the present data if one is prepared to assume on an ad hoc basis that the faces and their accompanying elaborations were well integrated. Unfortunately, the low recallability of the phrases found in Experiment 3 does not support the hypothesis that memory is redintegrative in the present case.

The data also are relevant to the issue raised by Bahrick (1979) regarding the effectiveness of contextual elaboration on recognition for target items never encountered before. Bahrick argued that recognition decisions regarding such unfamiliar items as the faces of strangers should be based mainly on item characteristics and not be influenced by contextual information. The four experiments reported here provide evidence to the contrary: An elaborated encoding context enhanced facial recognition in all four experiments.

With respect to the more general psychological question of whether visual recognition memory is affected by verbal factors, the present results give a positive answer. This finding is consistent with those of other researchers who have found a facilitative effect of verbal labeling on recognition memory for ambiguous or nonsensical visual stimuli (e.g., Ellis, 1968; Rafnel \& Klatzky, 1978). It is also consistent with Loftus and Kallman's (1979) finding that recognition memory for pictures of complex naturalistic scenes was markedly improved when subjects were asked "to write down the name of any detail in the picture that they thought might subsequently help in terms of recognizing the picture" (p. 202). Especially interesting is the encoding/ retrieval interaction found in this study. At test, Loftus and Kallman asked all of their subjects to write down any detail of the picture that they had used as the basis for making their yes-no recognition decision. When recognition was conditionalized on whether or not a detail was named at test, there was a pronounced reinstatement effect. That is, recognition was superior for the group that had looked for and named visual details at study, provided that the details also were named at test; for pictures that elicited no naming of details at test, encoding condition had no effect on recognition. This interaction between encoding and retrieval conditions, in which the target events are visual and elaboration is verbal, parallels our findings in Experiment 4 in spite of procedural differences between the studies. The salient differences are that, in the Loftus and Kallman study, specific visual details were generated by the subjects, whereas, in our study, nonvisual information was provided by the experimenters.
There remains the theoretical question about the nature of the elaboration process induced by the verbal statements in our experiments. One possible theoretical explanation, presented most clearly by Anderson (1980 Anderson \& Reder, 1979), is that verbal information and visual information are combined additively to yield a more extensive abstract memory code. The richer or more extensive the memory code associated with a visual stimulus, the more likely it is that it will be recognized. An alternative explanation, consistent with Gibson's (1969) theory of perceptual learning and similar to the position advanced by one of us (Winograd, 1981) in accounting for the facilitative effects on memory of certain kinds of judgments about faces, assumes that verbal information modifies visual information processing itself. To be concrete, this line of argument assumes that the role of the descriptive phrases is to induce a scan of the face; it is as if the subject examines the face to find an answer to the question "What kind of a man is this who likes Mozart?" Scanning the face not only allows more featural information to be encoded, it increases the likelihood that a distinctive feature of the face will be encoded. Loftus and Kallman (1979) and Winograd (1981) have provided evidence that distinctive features play a role in visual memory. The enhancement of recognition by reinstatement of the study context presents no problems to either account. The richer the retrieval environment, the larger is the number of propositions accessed, from the point of view of a propositional explanation of elaboration. For the distinctive visual feature position, reinstatement of context should make it more likely that the same scan is repeated and the same distinctive feature is sampled at retrieval as at test. The results of our experiments can be accommodated by either of these theoretical frameworks.

\section{REFERENCES}

Anderson, J. R. Cognitive psychology and its implications. San Francisco: Freeman, 1980.

Anderson, J. R., \& ReDER, L. M. An elaborative processing explanation of depth of processing. In L. S. Cermak \& F. I. M. Craik (Eds.), Levels of processing in human memory. Hillsdale, N.J: Erlbaum, 1979.

BAHRICK, H. P. Broader methods and narrower theories for memory research: Comments on the papers by Eysenck and Cermak. In L. S. Cermak \& F. I. M. Craik (Eds.), Levels of processing in human memory. Hillsdale, N.J: Erlbaum, 1979.

Chance, J., \& Goldstein, A. G. Recognition of faces and verbal labels. Bulletin of the Psychonomic Society, 1976, 7 , 384-386.

Clifton, C., \& Slowiacze K, M. L. Integrating new information with old knowledge. Memory \& Cognition, 1981, 9, 142-148.

CrAik, F. I. M., \& J ACOBY, L. L. Elaboration and distinctiveness in episodic memory. In L. Nilsson (Ed.), Perspectives on memory research. Hillsdale, N.J: Erlbaum, 1979.

Craik, F. I. M., \& Tulving, E. Depth of processing and the retention of words in episodic memory. Journal of Experimental Psychology: General, 1975, 104, 268-294.

ELLIS, H. Transfer of stimulus predifferentiation to shape recog- 
nition and identification learning: Role of properties of verbal labels. Journal of Experimental Psychology, 1968, 78, 401-409.

Fisher, R. P., \& Craik, F. I. M. The effects of elaboration on recognition memory. Memory \& Cognition, 1980, 8, 400-404.

Gibson, E. J. Principles of perceptual learning and development. New York: Appleton-Century-Crofts, 1969.

Goldstein, A. G., \& Chance, J. E. Visual recognition memory for complex configurations. Perception \& Psychophysics, 1970, 9, 237-241.

Klatzky, R. L., Martin, G. L., \& Kane, R. A. Semantic interpretation effects on memory for faces. Memory \& Cognition, 1982, 10, 195-206.

KLe IN, K., \& Saltz, E. Specifying the mechanisms in a levelsof-processing approach to memory. Journal of Experimental Psychology: Human Learning and Memory, 1976, 2, 671-679.

Loftus, G. R., \& Kallman, H. J. Encoding and use of detail information in picture recognition. Journal of Experimental Psychology: Human Learning and Memory, 1979, 5, 197-211.

RAFNel, K. J., \& KLATzKy, R. L. Meaningful-interpretation effects on codes of nonsense pictures. Journal of Experimental Psychology: Human Learning and Memory, 1978, 4, 631-646.
Reder, L. M., \& Ande rson, J. R. A partial resolution of the paradox of interference: The role of integrating knowledge. Cognitive Psychology, 1980, 12, 447-472.

Schulman, A. I. Memory for words recently classified. Memory \& Cognition, 1974, 2, 47-52.

Watkins, M. J., Ho, E., \& Tulving, E. Context effects in recognition memory for faces. Journal of Verbal Learning and Verbal Behavior, 1976, 15, 505-518.

Winograd, E. Encoding operations which facilitate memory for faces across the life span. In M. M. Gruneberg, P. E. Morris, \& R. N. Sykes (Eds.), Practical aspects of memory. New York: Academic Press, 1978.

WinogRAD, E. Elaboration and distinctiveness in memory for faces. Journal of Experimental Psychology: Human Learning and Memory, 1981, 7, 181-190.

Winograd, E., \& Rivers-Bulkeley, N. T. Effects of changing context on remembering faces. Journal of Experimental Psychology: Human Learning and Memory, 1977, 3, 397-405.

(Received for publication April 19, 1982; revision accepted August 3, 1982.) 